\title{
CrackLabel: A Thresholding-Based Crack Labeling Tool for Asphalt Pavement Images
}

\author{
Nor Aizam Muhamed Yusof ${ }^{1}$, Muhammad Khusairi Osman ${ }^{2, *}$, Fadzil Ahmad², Mohaiyedin Idris², \\ Anas Ibrahim ${ }^{3}$, Nooritawati Md Tahir ${ }^{4}$, Norbazlan Mohd Yusof ${ }^{5}$ \\ ${ }^{1}$ Department of Electrical Engineering, Politeknik Tuanku Sultanah Bahiyah, Kedah, Malaysia \\ ${ }^{2}$ Faculty of Electrical Engineering, Universiti Teknologi MARA, Cawangan Pulau Pinang, Malaysia \\ ${ }^{3}$ Faculty of Civil Engineering, Universiti Teknologi MARA, Cawangan Pulau Pinang, Malaysia \\ ${ }^{4}$ Faculty of Electrical Engineering, Universiti Teknologi MARA, Selangor, Malaysia \\ ${ }^{5}$ PLUS Berhad, Persada PLUS, Selangor, Malaysia
}

Received February 27, 2021; Revised July 27, 2021; Accepted August 9, 2021

\section{Cite This Paper in the following Citation Styles}

(a): [1] Nor Aizam Muhamed Yusof, Muhammad Khusairi Osman, Fadzil Ahmad, Mohaiyedin Idris, Anas Ibrahim, Nooritawati Md Tahir, Norbazlan Mohd Yusof, "CrackLabel: A Thresholding-Based Crack Labeling Tool for Asphalt Pavement Images," Civil Engineering and Architecture, Vol. 9, No. 5A, pp. 58 - 67, 2021. DOI: 10.13189/cea.2021.091307.

(b): Nor Aizam Muhamed Yusof, Muhammad Khusairi Osman, Fadzil Ahmad, Mohaiyedin Idris, Anas Ibrahim, Nooritawati Md Tahir, Norbazlan Mohd Yusof (2021). CrackLabel: A Thresholding-Based Crack Labeling Tool for Asphalt Pavement Images. Civil Engineering and Architecture, 9(5A), 58 - 67. DOI: 10.13189/cea.2021.091307.

Copyright $\odot 2021$ by authors, all rights reserved. Authors agree that this article remains permanently open access under the terms of the Creative Commons Attribution License 4.0 International License

\begin{abstract}
In an image classification system based on deep learning, a training dataset is a set of labelled images and is often composed of a large number of images. Image labelling tool is usually used to facilitate in creating the training dataset used by the classifier during the learning phase. This paper presents a new image labelling tool called CrackLabel that can automatically label the cracks in the asphalt pavement images. A specially designed image thresholding method called the Global and Lower Quartile Average Intensity (GLQAI) method is utilised. In this study, the training dataset is developed by using real pavement images that resized to $1024 \times 768$ resolution. First, crack images are automatically segmented into 768 small patches with $32 \times 32$ resolution (pixel). Then, a threshold-based method is applied to automatically segment these patches into two classes which are crack and non-crack patches. The image thresholding method based on the average of global average intensity (GAI) and lower quartile intensity (LQI), namely GLQAI is proposed for this task. Next, the labelling process is performed by assigning patches associated with the crack and background into the crack and non-crack folder, respectively. Finally, the performance of CrackLabel is benchmarked by comparing the results with the manual label crack images by human experts, and three commonly
\end{abstract}

used thresholding methods; Otsu, Kapur and Kittler-Illingworth thresholding. Experimental results show that the proposed thresholding method achieved the best classification rate among various thresholding methods with $94.50 \%, 93.60 \% 94.00 \%$ and $94.05 \%$ for recall, precision, accuracy, and F-score respectively. In conclusion, it is observed that the proposed method using the newly threshold algorithm is very effective in label images into the crack and non-crack patches to maximize the training performance.

Keywords Asphalt Pavement Assessment, Crack Detection, Image Labelling Tool, Image Threshold

\section{Introduction}

Manual inspection of pavement distress requires the surveyors to walk along the road to assess and record the information of pavement cracks. As road networks have kept growing in extension, this method will be closely related to labour-force resources, time-consuming and need some consideration with higher capital expenditures for maintenance cost [1], [2]. Furthermore, by using the 
manual inspection method, there is a lack of precise information about the road condition. This method completely depends on the surveyors' level of experience and knowledge that is expected to influence the subjectivity of human interpretation and perception of pavement rating [3][4] since the surveyors provide different result analyses. This method is also no longer efficient and produced a high human error rate in data collection.

Considering the negative trends of manual inspection, the current effort to automate pavement crack detection and classification by using image processing and artificial intelligence are actively conducted. The development of vision-based automated pavement crack detection methods such as intensity-thresholding [5][6], edge detection [7], wavelet transforms [8][9], and texture-analysis, [10][11] methods are well-documented in the literature. Hence, an automated system for pavement crack detection and classification has been developed to monitor and evaluate pavement conditions as a routine inspection [12].

The demand for automated assessment of road surfaces using a deep neural network (DNN) is ever-increasing [13]. One of the latest DNN techniques called deep convolution neural network (DCNN), has shown outstanding performance in many automated pavement assessment systems [14]-[17]. Few factors that improve the performance of DCNN of classifiers such as quality and large size of a dataset, selection of suitable training algorithms and combine the prediction from multiple models (ensemble method) [18]. Among them, providing quality and large size of the dataset to the network is the most important factor as the DCNN is a type of data-driven method. The network requires large datasets of labelled images to accurately model the problem and produce a high classification rate during training and testing phases [19]-[22].

Image annotation or labelling tool is usually used to ease the development of a training dataset for a DCNN to perform image recognition and computer vision tasks [23]. Recently, statistical techniques have been applied by many researchers to relate words and images [24][25]. In [25], Makadia and colleagues introduced an image labelling tool using a straightforward baseline technique that transfers keywords from the image. Their proposed baseline method combines the distance measures of global colour and texture. The experimental result shows the proposed method leads to better results against the existing labelling methods.

Duygulu et al. [26] proposed a machine translation based on an object recognition model. In this work, the object recognition process is achieved through labelling image regions with words. The images were partitioned into regions and subsequently classified into region types using the obtained colour and texture as features. The expectation-maximization (EM) method is used to map between region types and keywords supplied with the images. The method achieved $70 \%$ correct prediction on image labelling for object recognition.

According to Jeon et al. [27], a training dataset of images can be generated using an automated approach to annotate and retrieve images using a set of blob's vocabularies. The blobs are obtained from the image using the clustering method, and the cross-media relevance model is used to predict the word from the given blobs.

Similar to the other image recognition methods, detection of pavement crack using DCNN also required a large number of datasets. For example, research by Zhang and colleagues [28] used 30,000 images of each crack, sealed crack, and background to perform crack detection on road pavement. The dataset was developed by partitioning pavement images into small blocks or patches of $400 \times 400$ pixels in size by manually marked the centre square area of each block. The method was tested using 800 images with the dimension of $2,000 \times 4,000$ pixels. The proposed method has accurately distinguished cracks from sealed cracks and achieved satisfactory detection performance. However, the large size of the patch has influenced the speed of training which may slow down the crack detection process [29].

Another study conducted by $\mathrm{Li}$ et al., [30] has successfully built a model to classify pavement crack types using manually collected 28,000 small patches with $512 \times 512$ pixels in size. Before classification, the authors had resized the patches to $256 \times 256$ pixels to reduce computational effort. The large patch size for the training dataset would contribute to more parameters needed for the network and high computational burden [17].

In the analysis of a grid-scale image, Wang and $\mathrm{Hu}$ [31] proposed two databases by using two different grids of pixels $32 \times 32$ and $64 \times 64$ to segment 30,000 images for training set using 510 pavement images. However, their performance does not show a consistent result because their training dataset is not detailed enough. The experimental result showed that the $32 \times 32$ pixels have better performance than the $64 \times 64$ in precision. However, the $64 \times 64$ pixels have better performance in F-measure than $32 \times 32$.

Manual image labelling is a tedious and time-consuming process. To overcome this, there has been great interest in coming up with automatic ways to retrieve images based on image features [32][33]. Therefore, an automated image labelling for training dataset is the best solution that benefits to achieve good accuracy in network performance. Although the training datasets have publicly available for the classifier learning process, the problem of labelling remains since there are a lot of variables that differ from one camera setup to another [33]. Recent studies by [34][35] report that an automated image labelling could help to retrieved images in a large-scale dataset precisely and provides an alternative for the time-consuming work of manual image 
labelling.

There exist several image labelling tools [23]-[25], however, none of them was specifically designed for pavement crack detection. To the best of the authors' knowledge, the image labelling process for crack training datasets was conducted manually by researchers. No study has attempted to automate the labelling process of crack images. Motivated by the need of having large datasets that could be used for training classifiers, this study proposed a new image labelling tool called CrackLabel that can automatically label the cracks in the asphalt pavement images. A novel approach to segmenting crack and background patches using a new thresholding method called Global and Quartile Average Intensity (GQAI) is also introduced. Finally, the proposed image labelling tool is compared with human experts' manual labelling patches.

This paper is divided into four sections. Section 2 can be divided into several stages consisting of image acquisition, patch division and proposed threshold algorithm. The experimental result and performance evaluation are presented in Section III. Finally, Section IV concludes the research findings.

\section{Methods}

This section discusses in detail the proposed image labelling tool called CrackLabel using the Average and Lower Quartile Average Intensity (GLQAI) thresholding method. In general, the method comprises five (5) main steps which are image acquisition, patch division process; patch threshold and labelling process, evaluation of the experiment results and CrackLabel graphical user interface (GUI) development.

\subsection{Image Acquisition}

Image acquisition is the first part of preparing raw images to be processed. The images were taken along the road of Kulim, Kedah to Permatang Pauh, Penang using a hand-held NIKON digital camera with 16 Megapixels resolution. The images captured under daylight conditions with the camera lens vertical to the pavement surface. The distance of the camera from the pavement is approximately $80 \mathrm{~cm}$ to $100 \mathrm{~cm}$ with different variations of background caused by the presence of shadows, lighting effects, and non-uniform crack.
A total of 75 RGB images (35 transverse and 40 longitudinal cracks) with a resolution of $3456 \times 4608$ was captured using the digital camera. To reduce computation effort during the training process and proper patch division, all images were resized to the smaller resolution of $1024 \times 768$. Figure 1 shows an example of transverse and longitudinal cracks that had been captured during the image acquisition process.

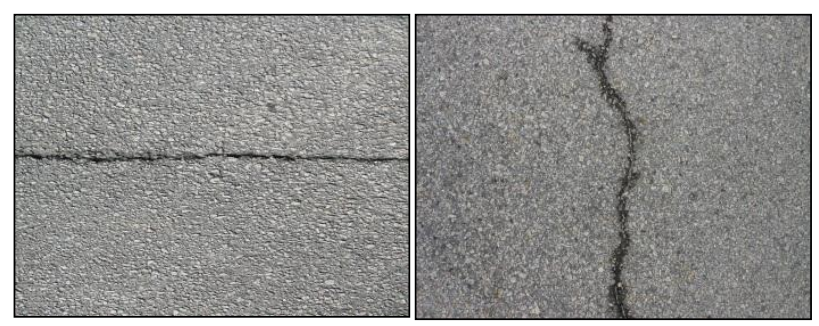

(a) Transverse crack

(b) Longitudinal crack

Figure 1. Example of asphalt pavement crack

\subsection{Patch Division}

The resized crack images are further segmented into small grids which are called patches. For this study, crack images were segmented into a small grid of $32 \times 32$ pixels without overlapping.

The system read the RGB image data vertically by $32 \times 32$ size non-overlapping and then move to the horizontal direction that obtains $32 \times 24$ grid block to create 768 image patches or blocks as illustrated in Figure 2. The images are stored in the memory matrix $32 \times 32$ dimensional with $24(\mathrm{x}) \times 32(\mathrm{y})$ arrays. Thus, the variable patch image begins at patches $(1,1)$ and ends at patches $(24,32)$. Figure 3 shows an example of a resized crack image and the result after applying patch division.

\subsection{Patch Thresholding and Labelling}

The purpose of image thresholding is to segment the patches into two types: crack and non-crack patches. In this study, a fully automated thresholding method called the Global and Lower Quartile Average Intensity (GLQAI) algorithm is proposed to find the optimal thresholding value for a given pavement crack image. The GLQAI algorithm consists of three (3) parameters which are Local Average Intensity (LAI), $\bar{x}_{L}$ and Global Average Intensity (GAI), 


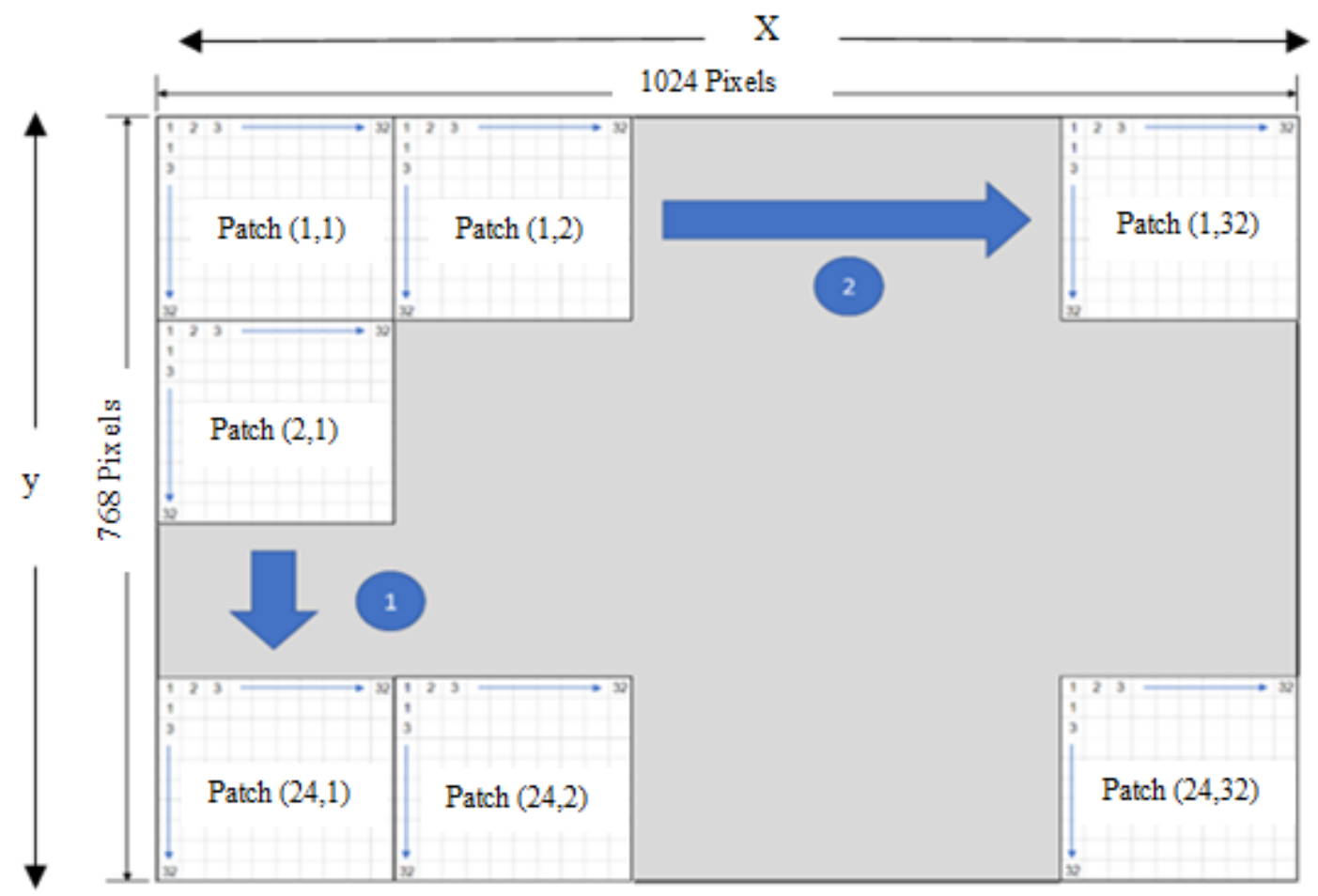

Figure 2. Illustration of patch divisional process

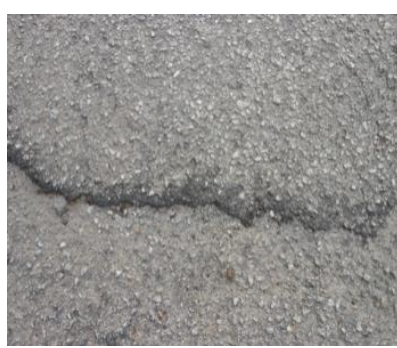

(a) RGB input image

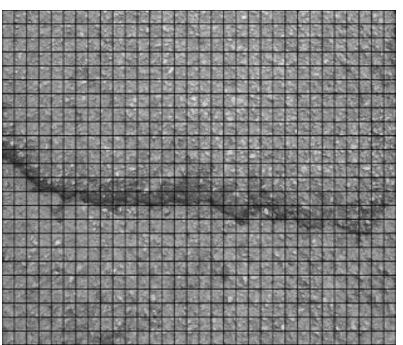

(b) $32 \times 32$ patch images

Figure 3. Example of crack image and its result after patch division process

$\bar{x}_{G}$ and lower quartile intensity (LQI). The LAI is obtained by calculating the average colour intensity within the patch. Therefore, each patch has its LAI value. Consider a crack image having $n$ number of patches. The LAI value of the $j$-th patch, $\bar{x}_{L, j}$ can be calculated using the following equation:

$$
\bar{x}_{L, j}=\frac{\sum_{i=1}^{m} I_{i}}{m}
$$

where $I_{i}$ and $m$ refer to the intensity value of $i$-th pixel and the total number of pixels in the patch. Meanwhile, the GAI, $\bar{x}_{G}$ is obtained by calculating the average colour intensity within the image. Therefore, each crack image will have the GAI value. The GAI, $\bar{x}_{G}$ can be calculated using (2),

$$
\bar{x}_{G}=\frac{\sum_{j=1}^{n} \bar{x}_{L, j}}{n}
$$

Cracks usually appear in the form of strong lines with low intensity. Our direct observation using several crack images has found that most of the crack patches will have a lower LAI value compared to the non-crack patches. Therefore, by manipulating LAI and GAI values, a simple thresholding method can be formulated to partition the patches into crack and non-crack as follows:

$$
P=\left\{\begin{array}{cc}
1 & \text { if } \bar{x}_{L, j} \leq \bar{x}_{G} \\
0 & \text { otherwise }
\end{array}\right\}
$$

where $\mathrm{P}$ is the patch class. A patch is labelled as a crack patch if $P=1$, while $P=0$ for the non-crack patch.

The simple thresholding method is highly dependent on the GAI value in separating the crack and non-crack patches. Typically, in a pavement crack image, the number of patches associated with crack is usually lower than the number of non-crack patches. Since the non-crack patches are usually associated with high intensity, the increasing number of these patches will indirectly increase the GAI value. This high GAI value will lead to the over-segmentation problem, whereby non-crack patches are incorrectly assigned as crack patches. To minimise the over-segmentation after applying the simple thresholding method, the lower quartile intensity (LQI) method is introduced. 
The quartile is a statistical term that refers to partitioning a dataset into four or more equal parts. The lower quartile, $Q_{L}$ is the middle value between the first and second quarter of the dataset. Figure 4 illustrates the quartile and the lower quartile of a dataset. By assuming that the crack patches are usually associated with the lower value of intensity, the LQI method takes the intensity at $Q_{L}$ value for the thresholding process. The method started by sorting the LAI values of a given crack image in ascending form. Then, the threshold value of LQI, $x_{Q_{L}}$ is obtained by finding the LAI value $Q_{L}$.

The LQI method fixed the threshold value at $25 \%$ of the overall LAI values. To make it adaptive, this study proposed a new thresholding method called Global and Lower Quartile Average Intensity (GLQAI) that takes into consideration both GAI and LQI threshold values. The GLQAI method determines the threshold value by averaging as formulated in (4),

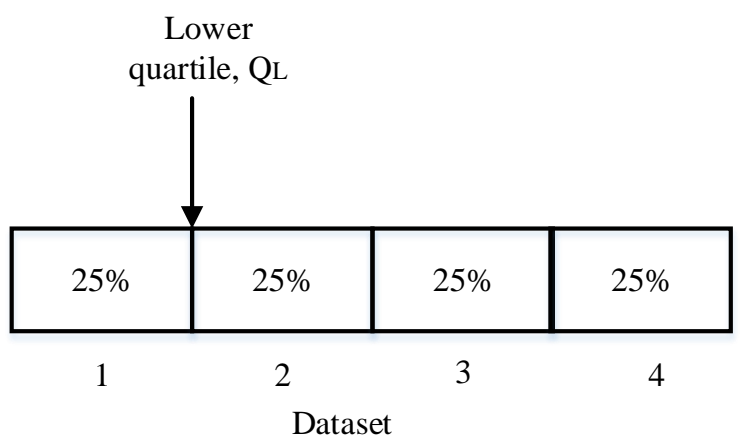

Figure 4. The quartile and lower quartile, $\mathrm{Q}_{\mathrm{L}}$

$$
P_{G L Q A I}=\left\{\begin{array}{l}
1 \quad \text { if } \quad \bar{x}_{L, j} \leq \frac{\bar{x}_{G}+x_{Q_{L}}}{2} \\
0 \quad \text { otherwise }
\end{array}\right.
$$

where $\mathrm{P}_{\mathrm{GLQAI}}$ is the patch class labelled using the GLQAI method. Similar to the previous method, a patch is labelled as a crack patch if $P=1$, while $P=0$ for the non-crack patch. Finally, the labelling process is performed by assigning patches associated with the crack and background into the crack and non-crack folders respectively. Figure 5 gives examples of crack and non-crack patches after applying the GLQAI method.

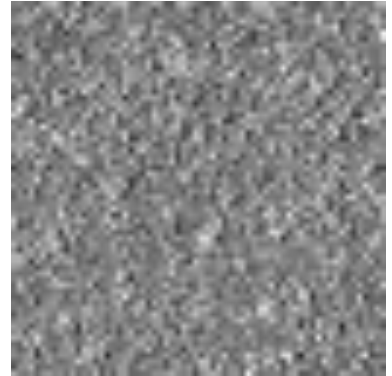

(a)

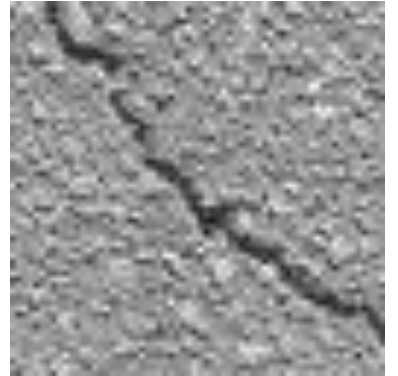

(b)
Figure 5. Example of (a) crack patch and (b) non-crack patch after applying GLQAI method.

\subsection{Evaluation of the Experimental Results}

Manual labelling of patches is considered as the gold standard in this study. The manual labelling process is achieved by manual visual examination and labelling of the patches by human experts. The result of the proposed GLQAI method is evaluated by comparing it with the manually labelling process and the performance was recorded. The performance was assessed using qualitative and quantitative evaluation. The qualitative evaluation is based on a visual inspection and scoring by three (3) human experts. Meanwhile, for quantitative evaluation, four (4) statistical evaluation metrics which are accuracy, precision, recall and $\mathrm{F}$ score are measured. The recall, precision, accuracy and $\mathrm{F}$ score are formulated as follows:

$$
\begin{gathered}
\text { Re call }=\frac{T P}{T P+F N} \\
\text { Precision }=\frac{T P}{(T P+F P)} \\
\text { Accuracy }=\frac{(T P+T N)}{(T P+T N+F P+F N)} \\
F \text { score }=\frac{2(\operatorname{Pr} \text { ecision } \times \operatorname{Re} \text { call })}{(\operatorname{Pr} \text { ecision }+\operatorname{Re} \text { call })}
\end{gathered}
$$

where $T P$ and $T N$ represent the total number of the crack and non-crack patches that the correctly classified. $F N$ and FP are the total numbers of crack and non-crack patches that are incorrectly classified by the threshold algorithm respectively.

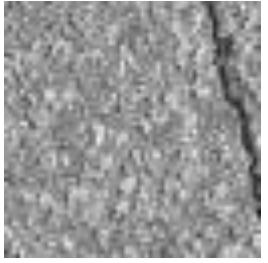

(i) $22 \%$

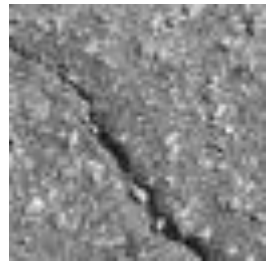

(ii) $25 \%$

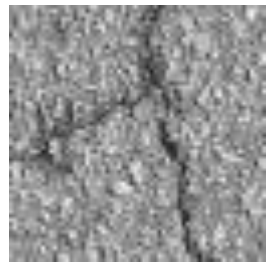

(iii) $28 \%$ (a) Crack patches with the percentage of pixels

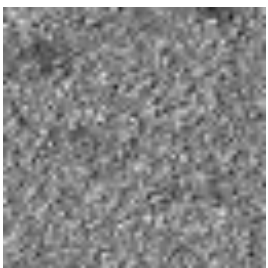

(i) $5 \%$

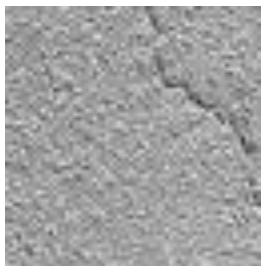

(ii) $10 \%$

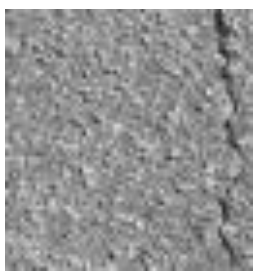

(iii) $15 \%$ (b) Non-crack patches with the percentage of pixels

Figure 6. Crack and non-crack patches obtained using Otsu thresholding

In addition to this, the study has also benchmarked the GLQAI method against three (3) commonly used thresholding methods which are Otsu [36], Kapur [37] and Kittler-Illingworth (K-I) thresholding methods [38]. However, the result of the GLQAI method cannot be directly compared with these three thresholding methods. This is attributed to the Otsu, Kapur and Kittler-Illingworth are pixel-based thresholding methods 
while the GLQAI is a patch-based thresholding method. To enable a direct comparison, firstly, the patch division process is applied to the resultant images of pixel-based thresholding methods. Then, the patch is labelled as a crack patch if the pixels belong to the crack occupies more than $20 \%$ of the total number of pixels in the patch. Figure 6 gives the example of crack and non-crack patches obtained using the Otsu thresholding method.

\subsection{Crack Labelling Tool using CrackLabel}

The final part of the automatic crack labelling process is to develop an image labelling tool that utilises the GLQAI method with menu-based or GUI. The image labelling tool is called the CrackLabel. The CrackLabel has accommodated almost all needed features for automatic crack labelling. The tool allows users to (1) select, load and display crack images; (2) automatically create patches; (3) perform automatic thresholding using the GLQAI method and (4) assign labels to all patches and save them into two different folders (crack or non-crack) at any specified location on the storage device, such as a hard disk or flash drive. The CrackLabel is developed using MATLAB. Figure 7 depicts the GUI of the CrackLabel.

\section{Results and Discussion}

The study conducts experimental evaluations on 75 images which comprise 35 transverse and 40 longitudinal crack images, as explained in the previous section. Both qualitative and quantitative evaluations have been used to evaluate the experimental results.

For qualitative evaluation, 15 transverse and 15 longitudinal crack images were randomly selected from the image dataset and their results using six (6) methods; GAI, LQI, GLQAI, Otsu, Kapur and K-I methods were presented to three (3) human experts.

On top of that, the image produced from the manual labelling process was also presented as a guideline. Figure 8 shows an example of the transverse crack image and its results using the manual labelling process and the six thresholding methods. Each expert was asked to rank these methods from best (rank 6) to worst (rank 1) according to manual labelling of patch images. The first-rank thresholding method score was obtained by averaging the ranking scores and selecting the method with the highest score.

Figure 9 compares the average score of different thresholding methods ranked by the experts for 30 crack images. The average score for the three experts is also presented. It can be seen that the GLQAI method is the best overall thresholding method. It significantly outperformed the other methods and obtained the highest scores for all experts with an average score of 5.8. The method produced images slightly similar to that of the manual labelling images, as can been seen in Figure 8(e). As a result, it became the most highly selected among the thresholding methods. Meanwhile, Otsu obtained the highest average score among the common thresholding method with 5.0, followed by Kapur (3.9) and K-I (3.1). On the contrary, the GAI method gives the lowest scores with an average of 1.0. The lowest score is associated with the under-segmentation problem that occurs in the GAI method, where most of the non-crack patches were incorrectly labelled as crack patches, as depicted in Figure 8(c). Similar to GAI, the LQI was unable to demonstrate satisfactory results.

The method achieved the second-lowest score with an average score of 2.2. The method produced results that suffer from over-segmentation, which causes the number of crack patches in the image to tend to be much smaller than the manual labelling image (See Figure 8(d)).

To further investigate the effectiveness of the proposed method, a quantitative evaluation was conducted on 75 images retrieved from the image acquisition process. As the patch size was set to $32 \times 32$, the study generated a total of 57,600 patches. The distribution of patches, according to their classes, is given in Table 1 . This distribution is obtained from images produced from the manual labelling process.

Table 1. Number of crack and non-crack patches

\begin{tabular}{|c|c|c|}
\hline Labelling process & Crack patches & Non-crack patches \\
\hline Manual & 2,123 & 55,477 \\
\hline
\end{tabular}

Manual labelling refers to a gold standard that serves as the benchmark for the best evaluation result from the experts. Therefore, recall, precision, accuracy and F-score are obtained from the comparison results of manual labelling against GAI, LQI, GLQAI, Otsu, Kapur and K-I methods. The study is also benchmarked with the previous thresholding method implemented on pavement crack as given in Table 2.

Table 2. Comparison of six image thresholding methods using statistical evaluation metrics

\begin{tabular}{|c|c|c|c|c|}
\hline Method & Recall & Precision & Accuracy & F-score \\
\hline Otsu & $89.00 \%$ & $83.50 \%$ & $85.50 \%$ & $86.15 \%$ \\
\hline Kapur & $84.80 \%$ & $83.10 \%$ & $83.30 \%$ & $83.94 \%$ \\
\hline $\begin{array}{c}\text { Kittler-Illing } \\
\text { worth }\end{array}$ & $84.40 \%$ & $81.50 \%$ & $83.30 \%$ & $82.92 \%$ \\
\hline GAI & $35.70 \%$ & $60.40 \%$ & $67.40 \%$ & $44.90 \%$ \\
\hline LQI & $72.05 \%$ & $71.95 \%$ & $72.81 \%$ & $71.99 \%$ \\
\hline GLQAI & $\mathbf{9 4 . 5 0 \%}$ & $\mathbf{9 3 . 6 0 \%}$ & $\mathbf{9 4 . 0 0 \%}$ & $\mathbf{9 4 . 0 5 \%}$ \\
\hline
\end{tabular}




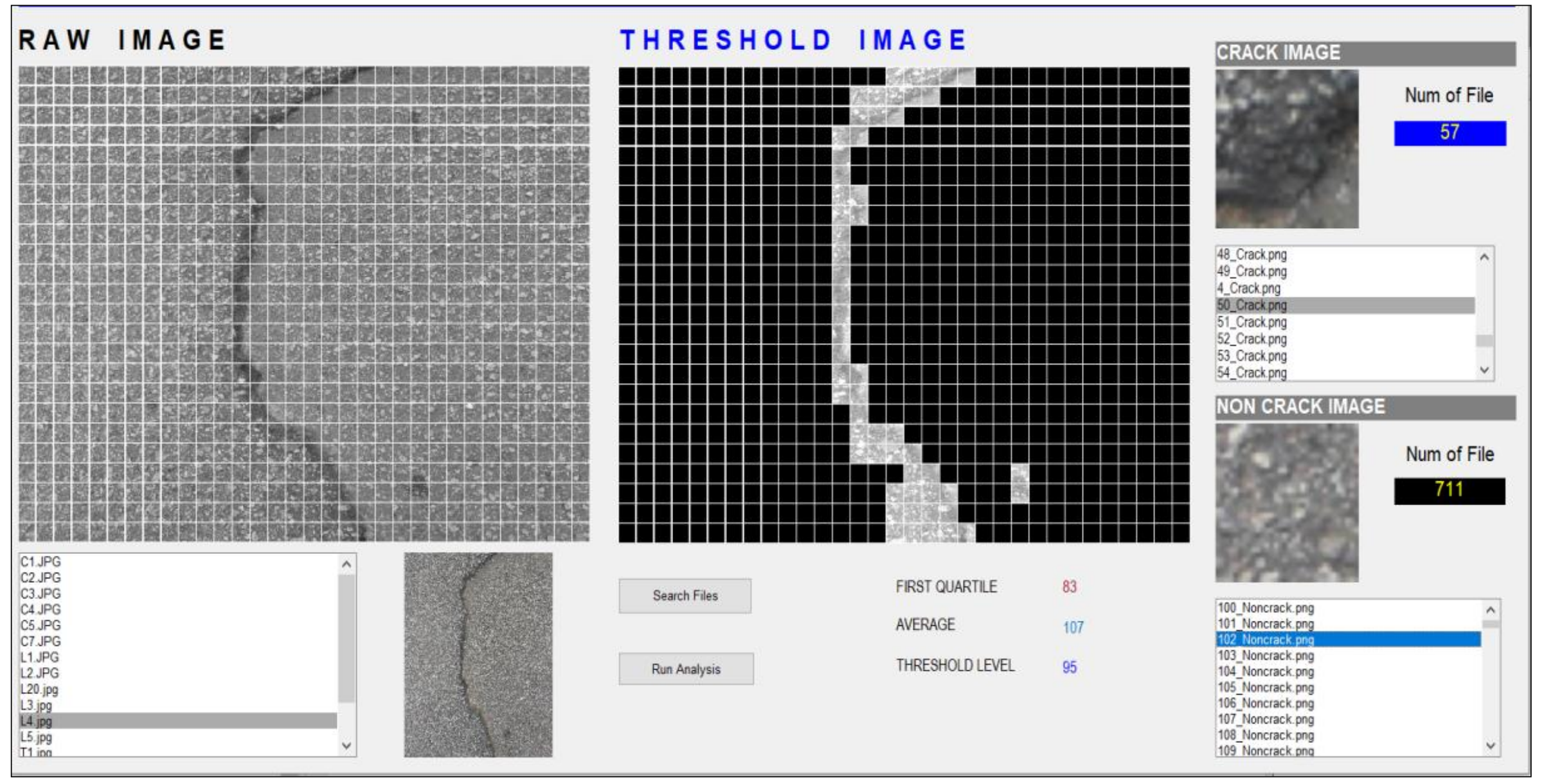

Figure 7. The CrackLabel tool 


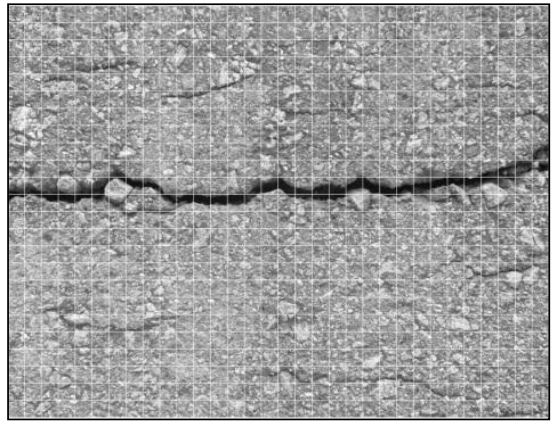

(a) Transverse crack image.

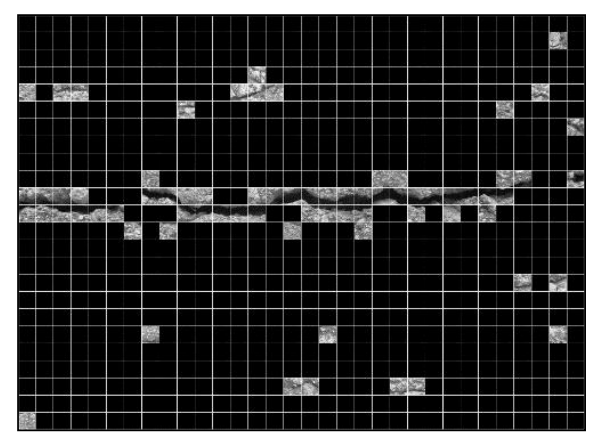

(e) GLQAI thresholding

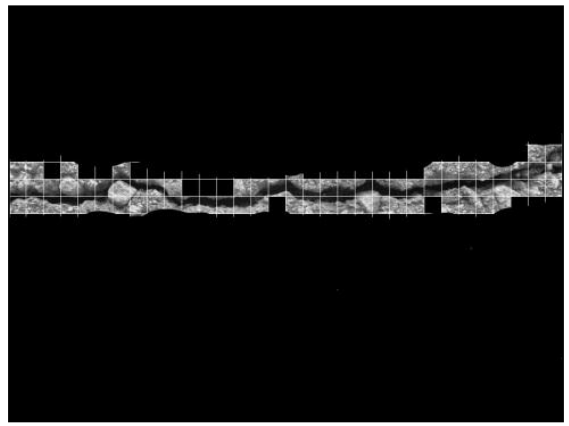

(b) Image produced from the manual labelling process.

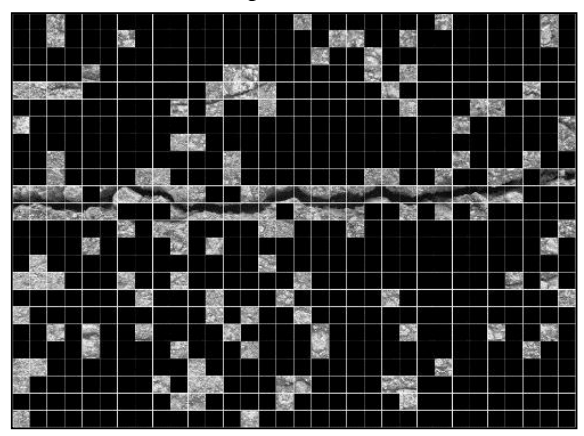

(e) Otsu thresholding

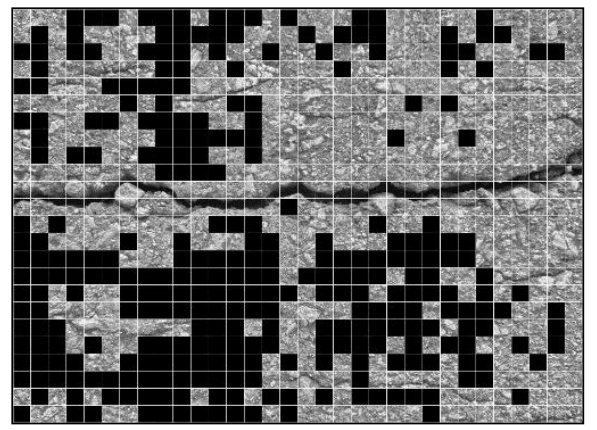

(c) GAI thresholding

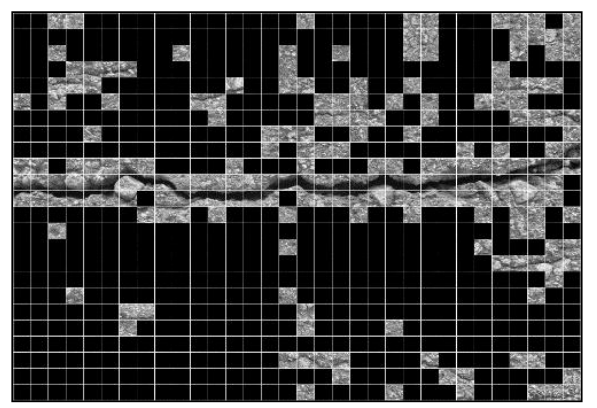

(f) Kapur thresholding

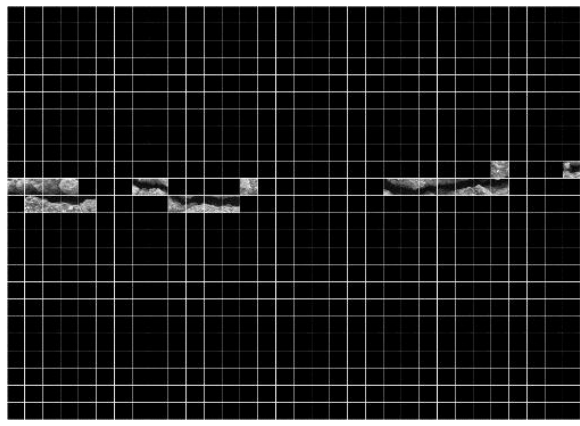

(d) LQI thresholding

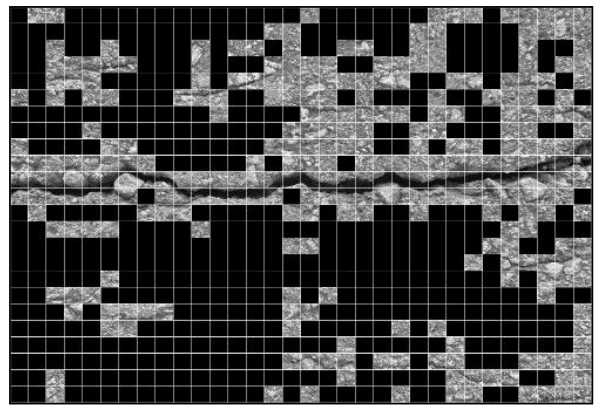

(g) Kittler-Illingworth thresholding

Figure 8. Example of transverse crack image and its image produced from manual labelling process and different thresholding methods

Based on the results, it is shown that both GAI and LQI produced the lowest detection score. Meanwhile, the performance achieved by Otsu thresholding is slightly higher among the common thresholding methods. The method achieved $89.00 \%, 83.50 \%$, $85.50 \%$ and $86.15 \%$ for recall, precision, accuracy and F-score respectively. It was also observed that the common thresholding methods are often difficult to distinguish rough pavement surfaces and thereby lower their precision. Overall, the GLQAI method demonstrated the best performance among all thresholding methods. The method achieved the highest crack detection score (recall $=94.50 \%$, precision $=93.60 \%$, accuracy $=94.00 \%$, F-score $=94.05 \%$. The proposed threshold method can effectively and efficiently extract the crack features in maximizing the crack identification from the

\section{pavement image.}

The proposed CrackLabel using the GLQAI method provides a solution to the manual labelling of crack images using a simple GUI application. The tool enables the user to select the desired image and perform crack labelling with a single click. The proposed CrackLabel tool was developed under MATLAB environment using a standard notebook with Intel Core i5-8250U CPU, 2GB RAM. It takes approximately 7 to 10 seconds to complete the labelling process for a crack image with the size of $1024 \times 768$. This processing time is significantly lower than the manual labelling process, which usually takes 3 to 5 minutes for the same image size. 


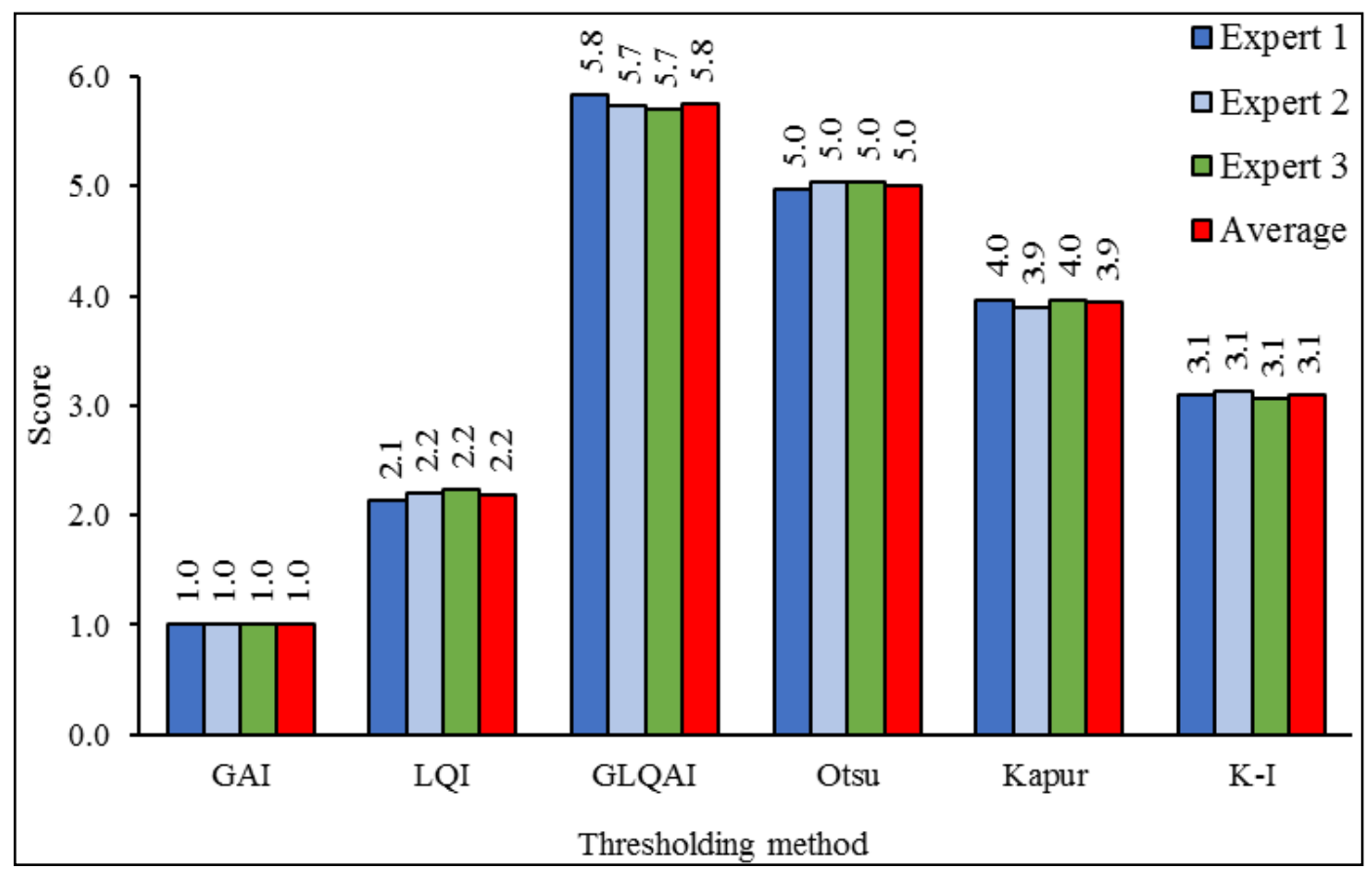

Figure 9. Example of transverse crack image and its image produced from manual labelling process and different thresholding methods

\section{Conclusions}

This study has successfully developed an image labelling tool called CrackLabel to ease the crack labelling process from asphalt pavement images. In addition, the study also introduced a new thresholding method called Global and Lower Quartile Average Intensity (GLQAI) to improve the detection of crack and non-crack patches in the image. The proposed method has outperformed other thresholding methods in qualitative and quantitative evaluations.

Despite the promising results reported so far, further research is required to enhance the crack detection performance. Future work will focus on integrating the GLQAI method with the manual labelling process. This semi-automated process will allow the user to remove or re-assign incorrect patch classes as the system still facing problems in the image consists of complex backgrounds.

\section{Acknowledgments}

The authors are grateful for the support and involvement of all parties, especially Universiti Teknologi MARA, Cawangan Pulau Pinang. This research is funded by the Fundamental Research Grant Scheme (FRGS), Grant No: FRGS/1/2019/TK04/UITM/02/30. The authors also appreciate the Ministry of Education (MOE) for providing Hadiah Latihan Persekutuan (HLP) scholarship.

\section{REFERENCES}

[1] M. Gavilán, D. Balcones, O. Marcos, D. F. Llorca, M. A. Sotelo, I. Parra, M. Ocaña, P. Aliseda, P. Yarza, and A. Amírola, "Adaptive Road Crack Detection System by Pavement Classification, Sensors, vol. 11, no. 10, pp. 9628 9657, Oct. 2011.

[2] B. Al-Mistarehi, GIS as a tool to Study Pavement distresses Distribution in Irbid city, Rev. Rom. Ing. Civila, vol. 5, no. 3, pp. 224-234, 2014.

[3] C. Koch, K. Georgieva, V. Kasireddy, B. Akinci, and P. Fieguth, A review on computer vision-based defect detection and condition assessment of concrete and asphalt civil infrastructure, Adv. Eng. Informatics, vol. 29, no. 2, pp. 196-210, 2015.

[4] L. Jing and Z. Aiqin, Pavement crack distress detection based on image analysis, 2010 Int. Conf. Mach. Vis. Human-Machine Interface, MVHI 2010, Kaifeng, China, no. c, pp. 576-579, 2010.

[5] S. Chambon and J-M. Moliard, Automatic road pavement assessment with image processing: Review and comparison, Sensors, vol. ISSN 1424-, no. 10, pp. 9628-9657, 2011.

[6] P. L. C. Henrique Oliveira, Automatic Road Crack Segmentation Using Entropy and Image Dynamic Thresholding, 17th Eur. Signal Process. Conf. (EUSIPCO 2009), vol. 63, no. 3, pp. 1066-1071, 2009.

[7] N. Attoh-Okine and A. Ayenu-Prah, Evaluating pavement cracks with bidimensional empirical mode decomposition, EURASIP J. Adv. Signal Process., vol. 2008, 2008.

[8] P. Subirats, J. Dumoulin, V. Legeay, and D. Barba, Automation of Pavement Surface Crack Detection using the Continuous Wavelet Transform, IEEE (ICIP 2006), vol. 1, no. 1, pp. 3037-3040, 2006.

[9] K. C. P. Wang, Q. Li, and W. Gong, Wavelet-based pavement distress image edge detection with a trous 
algorithm, Transp. Res. Rec., no. 2024, pp. 73-81, 2007.

[10] C. Koch, K. Georgieva, V. Kasireddy, B. Akinci, and P. Fieguth, A review on computer vision-based defect detection and condition assessment of concrete and asphalt civil infrastructure, Advanced Engineering Informatics. 2015.

[11] Y. Fujita, K. Shimada, M. Ichihara, and Y. Hamamoto, A method based on machine learning using hand-crafted features for crack detection from asphalt pavement surface images, Thirteen. Int. Conf. Qual. Control by Artif. Vis. 2017, vol. 10338, p. 103380I, 2017.

[12] T. B. J. Coenen and A. Golroo, A review on automated pavement distress detection methods, Cogent Eng., vol. 4, no. 1, pp. 1-23, 2017.

[13] Y. Sun, E. Salari, and E. Chou, Automated pavement distress detection using advanced image processing techniques, Proc. 2009 IEEE Int. Conf. Electro/Information Technol. EIT 2009, pp. 373-377, 2009.

[14] Z. Tong, J. Gao, Z. Han, and Z. Wang, Recognition of asphalt pavement crack length using deep convolutional neural networks, Road Mater. Pavement Des., no. ISSN: 1468-0629, pp. 1-16, 2017.

[15] G. Lin, A. Milan, C. Shen, and I. Reid, RefineNet: Multi-path refinement networks for high-resolution semantic segmentation, Proc. - 30th IEEE Conf. Comput. Vis. Pattern Recognition, CVPR 2017, vol. 2017-Janua, pp. 5168-5177, 2017.

[16] Y.-J. Cha, W. Choi, and O. Büyüköztürk, Deep Learning-Based Crack Damage Detection Using Convolutional Neural Networks, Comput. Civ. Infrastruct. Eng., vol. 32, no. 5, pp. 361-378, 2017.

[17] Z. Fan, Y. Wu, J. Lu, and W. Li, Automatic Pavement Crack Detection Based on Structured Prediction with the Convolutional Neural Network, Comput. Vis. Image Underst., pp. 1-9, 2018.

[18] K. Simonyan and A. Zisserman, Very Deep Convolutional Networks for Large-Scale Image Recognition, ICLR 2015, CA, USA, pp. 1-10, 7-9 May, 2015.

[19] G. Ghiasi and C. C. Fowlkes, Laplacian pyramid reconstruction and refinement for semantic segmentation, Lect. Notes Comput. Sci. vol. 9907 LNCS, pp. 519-534, 2016.

[20] M. Treml, J. A. Medina, T. Unterthiner, R. Dugresh, F. Firedmann, Speeding up Semantic Segmentation for Autonomous Driving, NIPS Work. 2016, pp. 1-7, 2016.

[21] C. L. Dihao Ai, Guiyuan Jiang, Lam Siew Kei, Automatic Pixel-Level Pavement Crack Detection Using Information of Multi-Scale Neighborhoods, IEEE Access, vol. 6, pp. 24452-24463, 2018.

[22] A. Zhang, K. Wang, Y. Fei, Y. Liu, C. Chen, G. Yang, Automated Pixel-Level Pavement Crack Detection on 3D Asphalt Surfaces with a Recurrent Neural Network, Comput. - Aided Civ. Infrastruct. Eng., vol. 00, pp. 1-17, 2018.

[23] J. Jeon and R. Manmatha, Using maximum entropy for automatic image annotation, Lect. Notes Comput. Sci. (including Subser. Lect. Notes Artif. Intell. Lect. Notes Bioinformatics), vol. 3115, pp. 24-32, 2004.
[24] D. M. Blei and M. I. Jordan, Modeling Annotated Data, Science (80), no. University of California Computer Science Division and Department of Statistics University of California, 2003.

[25] A. Makadia, V. Pavlovic, and S. Kumar, Baselines for image annotation, Int. J. Comput. Vis., vol. 90, no. 1, pp. 88-105, 2010.

[26] P. Duygulu, K. Barnard, J. F. G. de Freitas, and D. A. Forsyth, Object recognition as machine translation: Learning a lexicon for a fixed image vocabulary, Lecture Notes in Computer Science (including subseries Lecture Notes in Artificial Intelligence and Lecture Notes in Bioinformatics), vol. 2353. pp. 97-112, 2002.

[27] J. Jeon, V. Lavrenko, and R. Manmatha, Automatic Image Annotation and Retrieval using Cross-Media Relevance Models Categories and Subject Descriptors, Proc. 26th Int. ACM SIGIR Conf., pp. 119-126, 2003.

[28] K. Zhang, H. D. Cheng, B. Zhang, and D. Ph, Unified Approach to Pavement Crack and Sealed Crack Detection Using Preclassification Based on Transfer Learning, J. Comput. Civ. Eng., vol. 32, no. ISSN 0887-3801, pp. 1-12, 2018.

[29] A. Ahmadi, S. Khalesi, and M. Bagheri, Automatic road crack detection and classification using image processing techniques, machine learning and integrated models in urban areas: A novel image binarization technique, J. Ind. Syst. Eng., vol. 11, no. Iiec, pp. 85-97, 2018.

[30] B. Li, K. C. P. Wang, A. Zhang, E. Yang, and G. Wang, Automatic classification of pavement crack using deep convolutional neural network, Int. J. Pavement Eng., vol. 0, no. 0 , pp. $1-7,2018$

[31] X. Wang and Z. Hu, Grid-based Pavement Crack Analysis Using Deep Learning, in 2017 4th International Conference on Transportation Information and Safety, ICTIS 2017 Proceedings, 2017, pp. 917-924.

[32] V. Lavrenko, R. Manmatha, and J. Jeon, A model for learning the semantics of pictures, Adv. Neural Inf. Process. Syst., 2004.

[33] A. Petrovai, A. D. Costea, and S. Nedevschi, Semi-Automatic image annotation of street scenes, IEEE Intell. Veh. Symp. Proc., no. Iv, pp. 448-455, 2017.

[34] F. Wang, A survey on automatic image annotation and trends of the new age, Procedia Eng., vol. 23, pp. 434-438, 2011.

[35] M. Matsugu, K. Mori, Y. Mitari, and Y. Kaneda, Subject independent facial expression recognition with robust face detection using a convolutional neural network, Neural Networks, vol. 16, no. 5-6, pp. 555-559, 2003.

[36] Y. Chen, D. R. Chen, Y. Li, and L. Chen, Otsu's thresholding method based on gray level-gradient two-dimensional histogram, in CAR 2010 - 2010 2nd International Asia Conference on Informatics in Control, Automation and Robotics, 2010, vol. 3, pp. 282-285.

[37] W. Huang and N. Zhang, A Novel Road Crack Detection and Identification Method Using Digital Image Processing Techniques, Comput. Converg. Technol. (ICCCT), 7th Int Conf., pp. 397-400, 2012. 